\title{
Prevalence of Established Cardiovascular Disease in Patients with Type 2 Diabetes Mellitus in the UK
}

\author{
Dominik Lautsch · Tongtong Wang · Lingfeng Yang · Swapnil N. Rajpathak
}

Received: September 5, 2019 / Published online: October 4, 2019

(c) The Author(s) 2019

\section{ABSTRACT}

Introduction: The results of recently completed cardiovascular outcomes trials in patients with type 2 diabetes mellitus (T2DM) suggest that sodium-glucose cotransporter-2 (SGLT2) inhibitors and glucagon-like peptide (GLP) 1 receptor agonists have enhanced cardioprotective properties in patients with established cardiovascular disease (eCVD), but to a lesser degree in those without eCVD. SGLT2 inhibitors appear to be particularly beneficial in patients with heart failure. As recent data for the UK are lacking, we undertook to identify the percentage of T2DM patients with eCVD and heart failure in the UK.

Methods: This was a retrospective cohort study that utilized the Clinical Practice Research Datalink (CPRD) database in the UK. We included de-identified adult patients with T2DM with at least one encounter in the CPRD database between 1 January 2018 and 31 December 2018 in the analysis and extracted the full health records of these patients. eCVD was defined as myocardial infarction, stroke,

Enhanced Digital Features To view enhanced digital features for this article go to https://doi.org/10.6084/ m9.figshare.9873395.

D. Lautsch $(\varangle) \cdot$ T. Wang · L. Yang · S. N. Rajpathak Center for Observational and Real-World Evidence, Merck \& Co., Inc., Kenilworth, NJ, USA e-mail: dominik.lautsch@merck.com unstable angina pectoris, coronary artery disease and peripheral artery disease. We further assessed the number of patients with heart failure.

Results: From the total of 148,803 patients with T2DM analyzed (53\% were male; mean age was 65 years), 52,601 (35.4\%) suffered from eCVD and 8650 (5.8\%) suffered from heart failure $(73.7 \%$ of patients with heart failure overlap with those with atherothrombotic eCVD). Glycated hemoglobin levels of $<7 \%$ were attained by $49.5 \%$ of patients (with eCVD, 49.7\%; without eCVD, 49.3\%) ( $p<0.001)$.

Conclusion: Approximately one-third of patients with T2DM in the UK have concomitant CVD.

Funding: Merck Sharp \& Dohme Corp., a subsidiary Merck \& Co., Inc., Kenilworth, NJ, USA.

Keywords: Cardiovascular disease; Chronic kidney disease; Heart failure

\section{INTRODUCTION}

Diabetes mellitus is a chronic disease currently affecting an estimated 387 million people worldwide. The International Diabetes Federation estimates that by 2035 this number may rise to 592 million [1]. It is paramount to better understand the prevalence and associated complications of this disease, as well as the optimal treatment for patients with diabetes. 
Clinical trials conducted to date that have tested sodium-glucose cotransporter-2 (SGLT2) inhibitors and glucagon-like peptide-1 (GLP-1) receptor agonists, while heterogenous in design, have identified the potential of these drugs to reduce cardiovascular risk and improve blood glucose and glycated hemoglobin (HbA1c) control in patients with established cardiovascular disease (eCVD), but to a lesser degree in patients without CVD [2-6]. A recently completed trial, however, did not identify a difference of effect in patients with or without eCVD [7].

The 2018 joint consensus statement of the American Diabetes Association (ADA) and the European Association for the study of diabetes (EASD) therefore recommends the importance of individualizing management of a patient's T2DM based on the patient's profile, including comorbid conditions [8]. This current consensus paper recommends the initiation of SGLT2 inhibitors or GLP1 receptor agonists in patients with diabetes and concomitant CVD.

Given the results of recent cardiovascular outcomes trials and the current recommendations of the ADA and EASD, we conducted this study to define the percentage of T2DM patients with eCVD in the UK.

\section{METHODS}

In this retrospective cohort study, we analyzed patients with T2DM included in the UK Clinical Practice Research Datalink (CPRD) database [9]. The CPRD collects de-identified patient data from a network of general practitioner practices across the UK. In the CPRD, primary care data are linked to a range of other health-related data to provide a longitudinal, representative UK population health dataset. The data encompass over 35 million patients, including 11 million currently registered patients. We included all adult ( $\geq 18$ years of age on their index date) patients with T2DM with at least one encounter within the CPRD between 1 January 2018 and 31 December 2018 (index period). The index date for each patient was defined as the most recent encounter date during the 1-year index period. T2DM and comorbidities were assessed using all patient records available in the database prior to and including the index date. We used READ and SNOMED codes to identify T2DM and included those patients with at least two prescriptions for an oral diabetes medication. Patients with type 1 diabetes mellitus in any of their previous records were excluded.

Patients with T2DM and concomitant eCVD were categorized into two groups: (1) those with the atherothrombotic forms of CVD, defined as either myocardial infarction (MI), angina pectoris (AP), coronary artery disease (CAD), stroke, transient ischemic attack (TIA), or peripheral arterial disease (PAD); (2) those with heart failure. Concomitant atherothrombotic disease and heart failure was further identified and displayed utilizing a Venn diagram. We analyzed any potential differences between T2DM patients with concomitant eCVD and those without eCVD and compared the characteristics utilizing a Mann-Whitney $U$ test for calculated means of continuous variables and a Chi-square test for categorical variables. The patient variables included for analysis were age; gender; the most recent values of estimated glomerular filtration rate $\left(\mathrm{eGFR}, \mathrm{mL} / \mathrm{min} / \mathrm{m}^{2}\right.$ ) if directly available from documented records (no additional calculations to be performed); low-density lipoprotein-cholesterol $(\mathrm{mg} / \mathrm{dL})$ as available in the records and usually derived utilizing the Friedewald equation; systolic and diastolic blood pressure $(\mathrm{mmHg})$; and $\mathrm{HbA1c}(\%)$. We further assessed whether patients were ever smokers upon inclusion in the CPRD database. We consciously decided not to analyze the utilization of pharmacologic therapy (at class or compound level) as our aim was to conduct a purely epidemiological analysis on comorbidities.

The study was approved by the institutional document review committee and by UK Information Sharing and Analysis Center (ISAC; \#19_137R, July 2019). Patients provided informed consent to include their data in the CPRD, and any patient-related information was de-identified.

This study is based in part on data from the CPRD obtained under license from the UK Medicines and Healthcare products Regulatory Agency. The data are provided by patients and collected by the National Health Service as part 
of their care and support. The interpretation and conclusions contained in this study are those of the author/s alone.

\section{RESULTS}

A total of 148,803 patients with T2DM (male $53 \%$; mean age 65 years) were included in this analysis. Table 1 shows the patient characteristics. Of these 148,803 patients with T2DM, $52,601(35.4 \%)$ suffered from the atherothrombotic forms of eCVD and 8650 (5.8\%) from heart failure (Fig. 1). Patients considered to have eCVD in its atherothrombotic forms had a history of MI $(n=12,125 ; 8.2 \%)$, stroke or TIA $(n=14,709 ; 9.9 \%)$, unstable AP $(n=14,544$; $9.8 \%), \quad \mathrm{CAD} \quad(n=11,516 ; 7.7 \%)$ and $\mathrm{PAD}$ $(n=27,756 ; 18.7 \%)$.

Analysis of the age distribution of patients in the study showed that $40.8 \%$ were aged $<65$ years, $26.3 \%$ were aged $\geq 65$ and $<75$ years and $32.9 \%$ were aged $\geq 75$ years. We identified differences in the prevalence of the respective CVD entities according to age group, with a higher prevalence of disease at an older age (Table 2).

Laboratory results were available for 143,535 patients (96.4\%). Median HbA1c level was 7\% (interquartile range [IQR] 6.4-7.9 and 6.3-8.0\% for patients with and without eCVD, respectively; $p=0.047$ ). HbA1C levels of $<7 \%$ were achieved by $49.5 \%$ of patients ( 49.7 and $49.3 \%$ of patients with and without eCVD, respectively) $(p<0.001)$. The distribution of HbA1c levels was as follows: 25.5 and $24.9 \%$ of patients with and without eCVD, respectively, achieved HbA1c of 7.0 to $<8.0 \% ; 21.9$ and $11.9 \%$ of patients with and without eCVD, respectively, achieved HbA1c of $8.0<9.0 \%$; and 12.9 and $14.0 \%$ of patients with and without eCVD, respectively, achieved a HbA1c of $\geq 9.0 \%$.

The most recent eGFR rate was a median of 60 (IQR 53-76) and 68 (IQR 60-88) $\mathrm{mL} / \mathrm{min} /$ $1.73 \mathrm{~m}^{2}$ for patients with and without eCVD, respectively, $(p<0.001)$. Details on the distribution of chronic kidney disease according to the current National Kidney Foundation/Kidney Disease Outcomes Quality Initiative (K/DOQI) guidelines [10] are given in Fig. 2.

\section{DISCUSSION}

Our analysis showed that $35.4 \%$ of the patients with T2DM recorded in the CPRD database currently have eCVD, with $8.2 \%$ previously

Table 1 Demographic and clinical characteristics of patients

\begin{tabular}{lllr}
\hline Demographic and clinical characteristics & $\begin{array}{l}\text { With eCVD } \\
(\boldsymbol{N}=\mathbf{5 4 , 8 7 4})\end{array}$ & $\begin{array}{l}\text { Without eCVD } \\
(\boldsymbol{N}=\mathbf{9 3 , 9 2 9 )}\end{array}$ & $\boldsymbol{p}$ value \\
\hline Age (years; $n=148,803)$ & $73.5(12.1)$ & $62.9(15.0)$ & $<0.001$ \\
Male gender $(n=148,794)$ & $56.5 \%$ & $51.4 \%$ & $<0.001$ \\
eGFR $\left(\mathrm{ml} / \mathrm{min} / 1.73 \mathrm{~m}^{2}\right.$ body surface; & $60(53-76)$ & $68(60-88)$ & $<0.001$ \\
$\quad n=135,789)$ & & & \\
LDL-C $(\mathrm{mmol} / \mathrm{L} ; n=133,048)$ & $2.6(2.1)$ & $2.9(1.7)$ & $<0.001$ \\
SBP $(\mathrm{mmHg} ; n=147,945)$ & $130(120-140)$ & $132(122-140)$ & $<0.001$ \\
DBP $(\mathrm{mmHg} ; n=147,944)$ & $74(68-80)$ & $78(70-81)$ & $<0.001$ \\
HbAlc $(\% ; n=143,535)$ & $7(6.4-7.9)$ & $7(6.3-8)$ & 0.047 \\
BMI $\left(\mathrm{kg} / \mathrm{m}^{2}\right.$ body surface; $\left.n=144,208\right)$ & $29.7(26.1-34.1)$ & $30.5(26.8-35.2)$ & $<0.001$ \\
Smoking status: ever a smoker $(n=147,733)$ & $67.7 \%$ & $56.1 \%$ & $<0.001$ \\
\hline
\end{tabular}

$B M I$ Body mass index, $D B P$ diastolic blood pressure, eGFR estimated glomerular filtration rate, $H b A 1 c$ glycated hemoglobin, $L D L-C$ low-density lipoprotein cholesterol, $S B P$ systolic blood pressure 


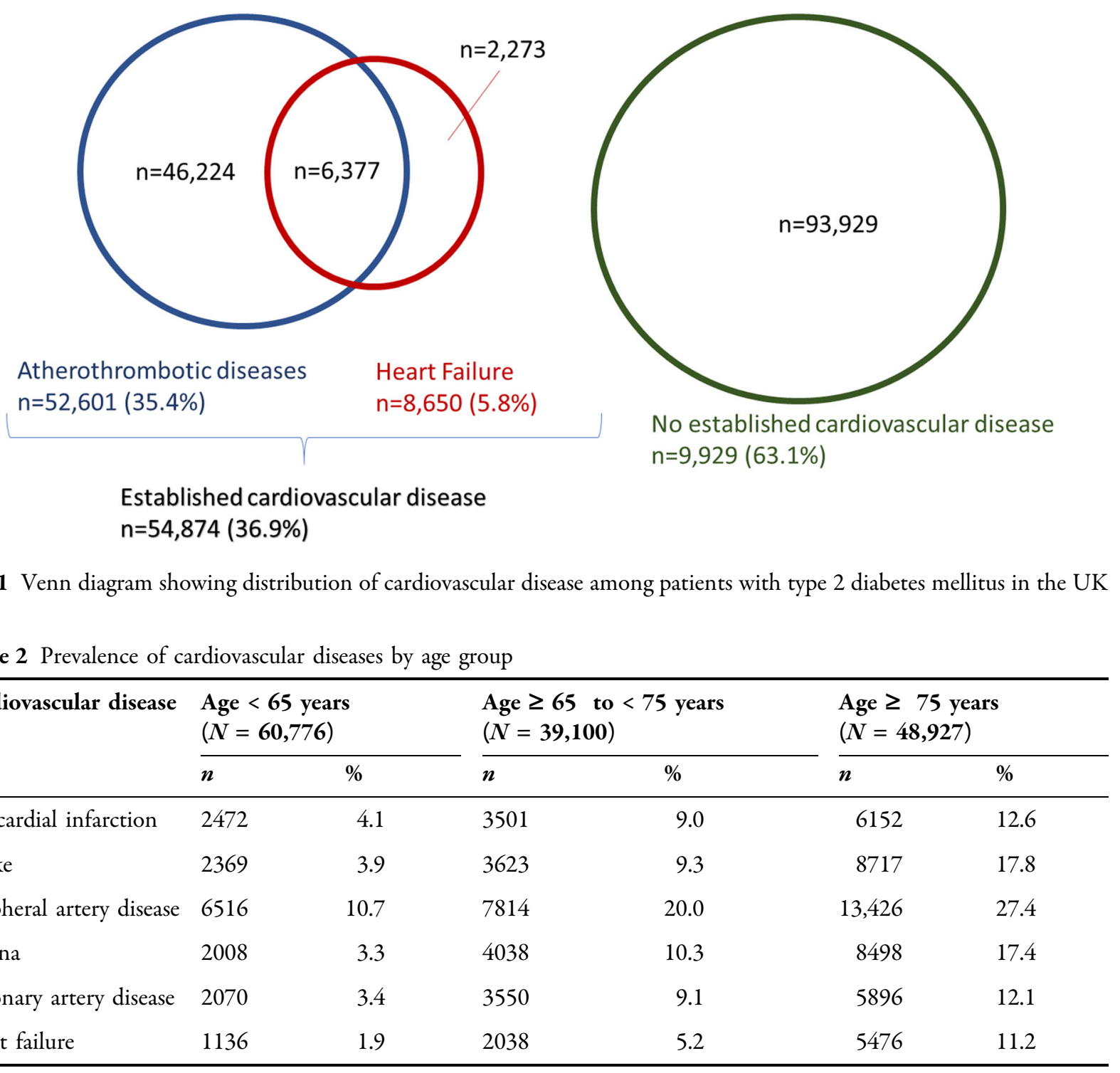

having had a MI and 9.9\% previously having had a stroke/TIA. We detected a different mean age for T2DM patients with and without eCVD, with those without eCVD having a mean age of 62.9 (standard deviation [SD] 15.0) years and those with eCVD having a mean age of 73.5 (SD 12.1) years. Older patients had a higher percentage of eCVD in all etiologies assessed. In patients aged $\geq 75$ years, $55.9 \%$ of the patients had eCVD, including heart failure, compared with $19.8 \%$ in patients aged $<65$ years. This difference was most pronounced for heart failure, with only $1.9 \%$ of patients younger than
65 years having heart failure compared with $11.2 \%$ (sixfold increased) of patients aged $\geq 75$ years. Heart failure can thus be considered a disease of the elderly, also in patients with concomitant diabetes.

A comparison of these data with those of other countries revealed a certain level of heterogeneity in the eCVD data depending on country analyzed, population examined and likely differences in coding. One study used US data collected on commercially insured patients, who are typically younger and thus healthier than the patients in the UK CPRD 


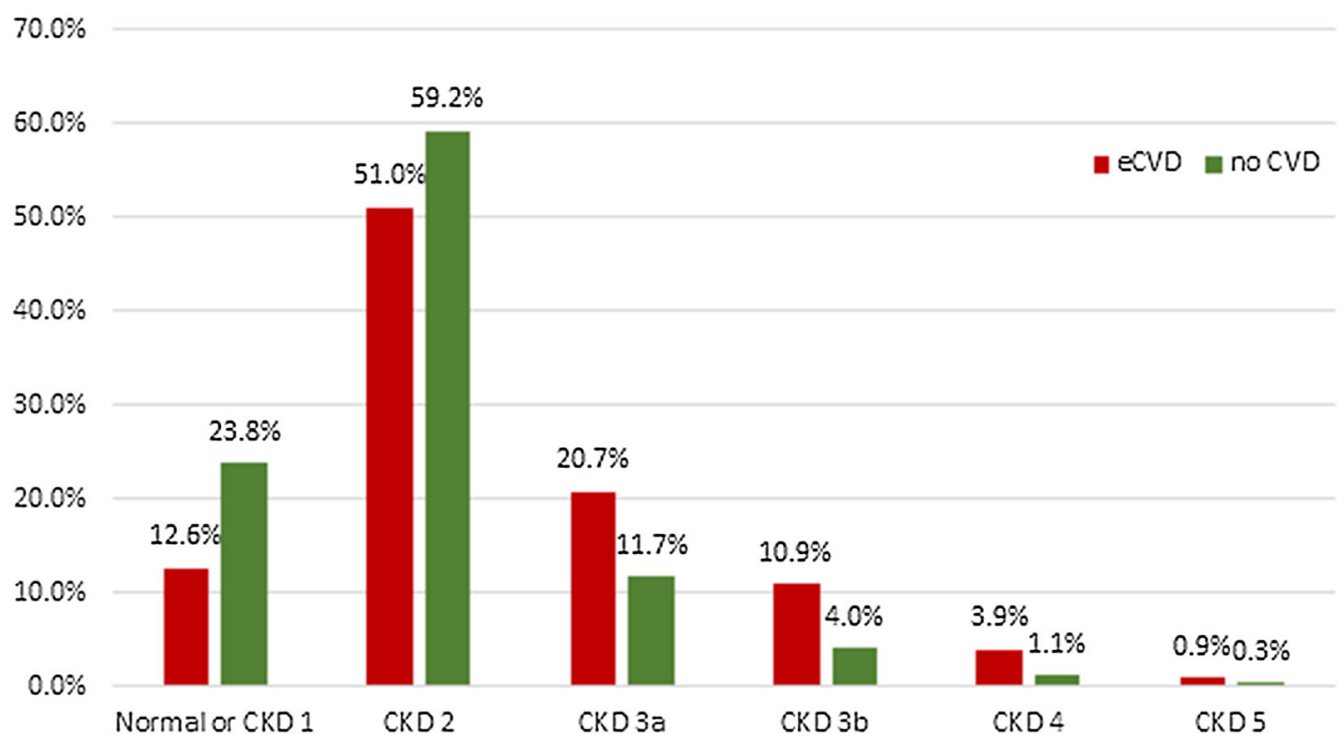

Fig. 2 Distribution of stages of chronic kidney disease (CKD) according to the National Kidney Foundation/ Kidney Disease Outcomes Quality Initiative (K/DOQI) based on guidelines on estimated glomerular filtration rate. eCVD Established cardiovascular disease. Normal or CKD

database as the former will enter the public Medicare program at age 65 years or upon retirement [11]. This review of commercially insured patients in the Quintiles Electronic Medical Record database (now known as the IQVIA database) identified an eCVD rate of $21.6 \%$ [11]. Recent analyses of data in the public health systems of Germany, the Netherlands, Norway and Sweden identified eCVD rates of $43.7,34.8,25.1$ and $31.4 \%$, respectively [12]. In this context, rates for MI ranged between 7.1 and $12.0 \%$, compared to $8.2 \%$ in our study in the UK. The rate of stroke was lowest in Norway, at $5.0 \%$, while all of the other countries, including the UK, ranged between 8.9 and $9.9 \%$. Interestingly, these studies showed marked differences in heart failure, with rates of $27.1,6.3,6.7,9.5,7.4$ and 5.8\% in Germany, the Netherlands, Norway, Sweden, SA and UK, respectively $[11,12]$.

Detailed knowledge of these rates is important since they can be used to inform treatment paradigms that are tailored to meet countryspecific needs. stage $1:>90 \mathrm{mLmin} / 1.73 \mathrm{~m}^{2}$, CKD stage $2: 60-89 \mathrm{~mL} /$ $\mathrm{min} / 1.73 \mathrm{~m}^{2}$; CKD stage 3a: $45-59 \mathrm{~mL} / \mathrm{min} / 1.73 \mathrm{~m}^{2}$; CKD stage $3 \mathrm{~b}: 30-44 \mathrm{~mL} / \mathrm{min} / 1.73 \mathrm{~m}^{2}$; CKD stage 4 : $15-29 \mathrm{~mL} / \mathrm{min} / 1.73 \mathrm{~m}^{2}$; CKD stage $5:<15 \mathrm{~mL} / \mathrm{min} /$ $1.73 \mathrm{~m}^{2}$

It is also of interest to understand how the results of cardiovascular outcomes trials with SGLT-2 inhibitors and GLP-1 receptor agonists apply to the general population of a country. Results from the CANVAS [3], DECLARE-TIMI 58 [6] and EMPA-REG OUTCOME [5] trials, all large, randomized, controlled cardiovascular outcome trials, suggest that underlying eCVD serves as an effect modifier [8]. A representative meta-analysis of these trials confirmed that the cardiovascular protective effect of SGLT2 inhibitors was only observed in patients with eCVD (hazard ratio [HR] 0.86, 95\% CI 0.80-0.93) versus those without eCVD (HR 1.00, 95\% CI $0.87-1.16$; $p$ for interaction $=0.05$ ) [13]. Within the class of GLP1 receptor agonists, a similar difference in outcomes between patients with eCVD and those without eCVD at baseline was shown in the LEADER study [2], which tested liraglutide ( $p$ for interaction $=0.04$ ), whereas no such difference was identified in the long-term study REWIND trial [7], which tested dulaglutide for up to 6 years. Both the SUSTAIN-6 [14] and PIONEER-6 [15] studies tested semaglutide, 
with inconclusive results due to either insufficient sample sizes or short duration of the trial.

As a general precaution, diabetes patients with eCVD should be closely monitored for control of their metabolic parameters and risk factors. Contemporary evidence from CPRD suggests that only $75 \%$ of patients (with or without diabetes) receive lipid-lowering therapy after a stroke [16]. Diabetes has been associated with a slightly higher rate of statin utilization. This figure needs to be compared to the $47 \%$ of overall diabetes patients in CPRD that were administered statins [17].

\section{Limitations}

In this observational, retrospective study, we report data as collected in the real world in the UK. There was no central laboratory or standard measure as to how the individual patients' results were retrieved. Some values were missing, and a reporting bias for some diagnoses in terms of higher age versus younger age or for patients who already manifest one disease entity versus those who have none cannot be excluded. However, our findings well reflect standard practice in the UK and mirror what physicians identify and record. We did not impute or correct for missing values, nor did we apply any regression to the mean. The patients included in our study were those included in the CPRD dataset. CPRD does not have appropriate level of detail in the READ and SNOMED codes to further distinguish with confidence heart failure patients with reduced ejection fraction from those with preserved ejection fraction.

\section{CONCLUSION}

Approximately one-third (35.4\%) of T2DM patients in the UK have concomitant atherothrombotic CVD. The prevalence of CVD is markedly higher in T2DM patients of older age.

\section{ACKNOWLEDGEMENTS}

Funding. This study and the Rapid Service Fee were funded by Merck Sharp \& Dohme Corp., a subsidiary of Merck \& Co., Inc., Kenilworth, NJ, USA.

Authorship. All named authors meet the International Committee of Medical Journal Editors (ICMJE) criteria for authorship for this article, take responsibility for the integrity of the work as a whole, and have given their approval for this version to be published.

Authorship Contributions. During the study, all authors contributed significantly. DL, TW and LY analyzed and interpreted the data. LY performed the statistical analyses. DL drafted the manuscript. SR critically contributed to the interpretation of the data and revised the draft.

Disclosures. Dominik Lautsch is an employee of Merck Sharp \& Dohme Corp., a subsidiary of Merck \& Co., Inc., Kenilworth, NJ, USA and holds restricted stock units of Merck \& Co., Inc., Kenilworth, NJ, USA. Tongtong Wang is an employee of Merck Sharp \& Dohme Corp., a subsidiary of Merck \& Co., Inc., Kenilworth, NJ, USA and holds restricted stock units of Merck \& Co., Inc., Kenilworth, NJ, USA. Lingfeng Yang is an employee of Merck Sharp \& Dohme Corp., a subsidiary of Merck \& Co., Inc., Kenilworth, NJ, USA and holds restricted stock units of Merck \& Co., Inc., Kenilworth, NJ, USA. Swapnil N. Rajpathak is an employee of Merck Sharp \& Dohme Corp., a subsidiary of Merck \& Co., Inc., Kenilworth, NJ, USA and holds restricted stock units of Merck \& Co., Inc., Kenilworth, NJ, USA.

Compliance with Ethics Guidelines. The study was approved by the institutional document review committee and by UK ISAC (\#19_137R, July 2019). Patients provided informed consent to inclusion of their data in CPRD. Any patient-related information was deidentified. 
Data Availability. All data generated or analyzed during this study are included in this published article.

Open Access. This article is distributed under the terms of the Creative Commons Attribution-NonCommercial 4.0 International License (http://creativecommons.org/licenses/ by-nc/4.0/), which permits any noncommercial use, distribution, and reproduction in any medium, provided you give appropriate credit to the original author(s) and the source, provide a link to the Creative Commons license, and indicate if changes were made.

\section{REFERENCES}

1. International Diabetes Federation. IDF diabetes atlas-8th edition. https://diabetesatlas.org/. Accessed 31 July 2019.

2. Marso SP, Daniels GH, Brown-Frandsen K, et al. Liraglutide and cardiovascular outcomes in type 2 diabetes. N Engl J Med. 2016;375:311-22.

3. Mahaffey KW, Neal B, Perkovic V, et al. Canagliflozin for primary and secondary prevention of cardiovascular events: results from the CANVAS Program (canagliflozin cardiovascular assessment study). Circulation. 2018;137:323-34.

4. Neal B, Perkovic V, Mahaffey KW, et al. Canagliflozin and cardiovascular and renal events in type 2 diabetes. N Engl J Med. 2017;377(7):644-57.

5. Zinman B, Wanner C, Lachin JM, et al. Empagliflozin, cardiovascular outcomes, and mortality in type 2 diabetes. N Engl J Med. 2015;373:2117-28.

6. Wiviott SD, Raz I, Bonaca MP, et al. Dapagliflozin and cardiovascular outcomes in type 2 diabetes. N Engl J Med. 2019;380(4):347-57.

7. Gerstein HC, Colhoun HM, Dagenais GR, et al. Dulaglutide and cardiovascular outcomes in type 2 diabetes (REWIND): a double-blind, randomised placebo-controlled trial. Lancet. 2019;394:121-30.
8. Davies MJ, D'Alessio DA, Fradkin J, et al. Management of hyperglycemia in type 2 diabetes, 2018. A consensus report by the American Diabetes Association (ADA) and the European Association for the Study of Diabetes (EASD). Diabetes Care. 2018;41(12):2669-701.

9. Herrett E, Gallagher AM, Bhaskaran K, et al. Data resource profile: clinical practice research datalink (CPRD). Int J Epidemiol. 2015;44(3):827-36.

10. National Kidney Foundation. K/DOQI clinical practice guidelines for chronic kidney disease: evaluation, classification, and stratification. Am J Kidney Dis. 2002;39[2 Suppl 1]:S1-266.

11. Iglay K, Hannachi H, Joseph Howie P, et al. Prevalence and co-prevalence of comorbidities among patients with type 2 diabetes mellitus. Curr Med Res Opin. 2016;32(7):1243-52.

12. Birkeland KI, Bodegard J, Norhammar A, et al. How representative of a general type 2 diabetes population are patients included in cardiovascular outcome trials with SGLT2 inhibitors? A large European observational study. Diabetes Obes Metab. 2018. https://doi.org/10.1111/dom.13612.

13. Zelniker TA, Wiviott SD, Raz I, et al. SGLT2 inhibitors for primary and secondary prevention of cardiovascular and renal outcomes in type 2 diabetes: a systematic review and meta-analysis of cardiovascular outcome trials. Lancet. 2019;393(10166):31-9.

14. Marso SP, Bain SC, Consoli A, et al. Semaglutide and cardiovascular outcomes in patients with type 2 diabetes. N Engl J Med. 2016;375:1834-44.

15. Husain M, Birkenfeld AL, Donsmark M, et al. Oral semaglutide and cardiovascular outcomes in patients with type 2 diabetes. $\mathrm{N}$ Engl J Med. 2019;381(9):841-51.

16. Yang Z, Edwards D, Massou E, Saunders CL, Brayne C, Mant J. Statin use and high-dose statin use after ischemic stroke in the UK: a retrospective cohort study. Clin Epidemiol. 2019;11:495-508.

17. Joshi R, Venkatesan S, Myles PR. A UK general practice population cohort study investigating the association between lipid lowering drugs and 30-day mortality following medically attended acute respiratory illness. PeerJ. 2016;4:e1902. https://doi.org/10.7717/peerj.1902. 